\title{
Allogeneic hematopoietic stem cell transplantation for T-prolymphocytic leukemia: a report from the French society for stem cell transplantation (SFGM-TC)
}

Thierry Guillaume ${ }^{1}, Y_{v e s}$ Beguin ${ }^{2}$, Reza Tabrizi ${ }^{3}$, Stéphanie Nguyen $^{4}$, Didier Blaise ${ }^{5}$, Eric Deconinck ${ }^{6}$, Rabah Redjoul ${ }^{7}$, Jérôme Cornillon ${ }^{8}$, Gaëlle Guillerm ${ }^{9}$, Nathalie Contentin ${ }^{10}$, Anne Sirvent ${ }^{11}$, Pascal Turlure ${ }^{12}$, Alexandra Salmon ${ }^{13}$, Anne Huynh ${ }^{14}$, Sylvie François ${ }^{15}$, Régis Peffault de Latour ${ }^{16}$, Ibrahim Yakoub-Agha ${ }^{17}$, Mohamad Mohty ${ }^{18}$

${ }^{1} \mathrm{CHU}$ Hôtel Dieu, Nantes, France; ${ }^{2} \mathrm{CHU}$ of Liège, Liège, Belgium; ${ }^{3}$ Hôpital Haut-Levêque, Pessac; ${ }^{4}$ La Pitié Salpétrière, Paris; ${ }^{5}$ nnstitut PaoliCalmettes, Marseille; ${ }^{6}$ Hôpital Jean Minjoz, Besançon; ${ }^{7}$ Hôpital Henri Mondor, Créteil; ${ }^{8} \mathrm{CHU}$ Saint Etienne, Saint-Etienne; ${ }^{9}$ Hôpital AugustinMorvan, Brest; ${ }^{10}$ Centre Henri Becquerel, Rouen; ${ }^{11} \mathrm{CHU}$ Lapeyronie, Montpellier; ${ }^{12} \mathrm{CHRU}$ Limoges, Limoges; ${ }^{13}$ Hôpital d'Enfants, Vandoeuvre; ${ }^{14} \mathrm{CHU}$ Toulouse, Toulouse; ${ }^{15} \mathrm{CHU}$ Angers, Angers; ${ }^{16} \mathrm{Hôpital}$ Saint-Louis, Paris; ${ }^{17} \mathrm{CHU}$ Lille, Lille; ${ }^{18}$ Hôpital Saint-Antoine, Paris, France

\begin{abstract}
T-prolymphocytic leukemia (T-PLL), a rare aggressive mature T-cell disorder, remains frequently resistant to conventional chemotherapy. Studies have suggested that allogeneic hematopoietic stem cell transplantation (HSCT) might possibly serve to consolidate the response to initial chemotherapy. The current report summarizes the outcome of 27 T-PLL cases identified in the registry in French Society for stem cell transplantation (SFGM-TC). Prior to HSCT, 14 patients were in complete remission (CR), 10 in partial response, three refractory, or in progression. Following HSCT, 21 patients achieved CR as best response. With a median follow-up for surviving patients of 33 (range, 6-103) months, 10 patients are still alive in continuous CR. Overall survival and progression-free survival estimates at $3 \mathrm{yr}$ were $36 \%(95 \% \mathrm{Cl}$ : 17-54\%) and 26\% (95\% Cl: 14-45\%), respectively. The relapse incidence after HSCT was 47\% occurring at a median of 11.7 (range, 2-24) months. Overall cumulative incidence of transplant-related mortality was $31 \%$ at $3 \mathrm{yr}$. These results suggest that HSCT may allow long-term survival in patients with T-PLL following induction treatment; however, it is associated with a significant rate of toxicity.
\end{abstract}

Key words T-prolymphocytic leukemia; allogeneic transplantation; chronic leukemia

Correspondence Thierry Guillaume, MD, Service d'Hématologie Clinique, CHU Hôtel-Dieu, 1 Place Ricordeau, 44093 Nantes Cedex, France. Tel: (33) 2400832 71; Fax: (33) 2400832 50; e-mail: thierry.guillaume@chu-nantes.fr

Accepted for publication 4 August 2014

doi:10.1111/ejh.12430

T-prolymphocytic leukemia (T-PLL) is a rare aggressive mature T-cell disorder with distinctive clinical, morphologic, immunophenotypic, and cytogenetic characteristics (1, 2). Indolent forms of the disease with moderate and isolated lymphocytosis have been described, but they usually progress to aggressive forms months or years later (3). Two proto-oncogenes TCL1a (14q32.1) and MTCP1 (Xq28) are involved in the pathogenesis of T-PLL while deletions or mutations of ataxia telangiectasia mutation in 11q23 have been involved in the sporadic and AT-associated T-PLL. T-PLL remains frequently resistant to conventional therapy. Median overall survival (OS) is approximately 7 months with such chemotherapy. The use of the anti-CD52 monoclonal antibody alemtuzumab could improve overall response rate $(4,5)$. Alemtuzumab given intravenously as first-line treatment resulted in an overall response rate of $91 \%$ with $81 \%$ completed response (6). More recently, Hopfinger et al., (7) used Fludarabine, mitoxantrone, and cyclophosphamide induction followed by alemtuzumab consolidation (FMC-A). For those who received the consolidation, the overall response rate reached $92 \%$, with median OS of 17.1 months and median progression-free survival (PFS) of 11.9 months. In an attempt to improve survival, isolated case reports have suggested that allogeneic hematopoietic stem cell transplantation 
(HSCT) may serve to consolidate the response to initial chemo-immunotherapy in T-PLL (8-14). The present report, we analyze the outcome of 27 T-PLL patients who received HSCT and were reported to the SFGM-TC registry.

\section{Patients and methods}

The current retrospective analysis includes 27 patients fulfilling the criteria for T-PLL (1) who underwent HSCT and had been reported to the registry of the SFGM-TC between 2000 and 2013. Patients were treated in 17 French and Belgian centers. This study was approved by the scientific committee of the SFGM-TC and conducted according to the principle of the Declaration of Helsinki. For the purpose of this analysis, a specific case report form was designed and centers were required to provide full diagnosis, transplant, and outcome data.

The analysis assessed pre- and post-transplant disease response, OS, PFS, transplant-related mortality (TRM), disease progression, and cumulative incidences of acute and chronic graft-versus-host disease (GVHD). OS and PFS were estimated using the Kaplan-Meyer method, while cumulative incidence rates were used for progression, TRM, and GVHD incidences with death or relapse treated as competing events. Potential prognostic factors including age, conditioning regimen (myeloablative vs. reduced-intensity regimen), total body irradiation (TBI) use as part of conditioning, type of donor (sibling vs. unrelated donor), graft source (peripheral blood stem cell vs. bone marrow or cord blood), stage of disease at transplantation, presence of acute or chronic GVHD were studied in univariate analyses. The log-rank test was used for univariate comparisons (GRAPHPAD software, La Jolla, CA, USA). Given the small number of patients, multivariate analysis was not performed.

\section{Results}

Patients' characteristics are summarized in Table 1. Prior to HSCT, 14 patients were in complete (CR), 10 in partial response (PR), three refractory, or in progression. One patient who received a second transplant was in PR prior to the second transplantation. Alemtuzumab was used in 17 patients prior to transplant (14 as first line, reused as second-line therapy for one patient, and as third line for three patients), fludarabine was used in four patients (two as first line and reused with associated with cyclophosphamide for one patient as second line, two patients received fludarabine associated with mitoxantrone and cyclophosphamide as second line and third line), and pentostatin was used in seven patients (two as first line or 5 second line). A CHOP-like regimen was used in 10 patients (eight as first line, two as second line and reused as second line for one patient). Alemtuzumab was administered subcutaneously for all patients.
Table 1 Patient, disease, and transplant characteristics

\begin{tabular}{|c|c|}
\hline Patient, $N$ & 27 \\
\hline Transplants, $N$ & $28^{1}$ \\
\hline Median age at transplant (yr, range) & $53(36-65)$ \\
\hline Male/female ratio & $17 / 10$ \\
\hline $\begin{array}{l}\text { Median time from diagnosis to } \\
\text { transplant, months (range) }\end{array}$ & $8.5(4.5-59)$ \\
\hline \multicolumn{2}{|l|}{ Pretransplant chemotherapy lines } \\
\hline 1 line & 13 \\
\hline 2 lines & 9 \\
\hline 3 lines & 4 \\
\hline 4 lines & 1 \\
\hline \multicolumn{2}{|l|}{ Clinical features } \\
\hline Splenomegaly & 9 \\
\hline Pleural effusion & 1 \\
\hline Cutaneous involvement & 4 \\
\hline \multicolumn{2}{|l|}{ Cytogenetics } \\
\hline Normal & 5 \\
\hline Abnormal & 12 \\
\hline Not done or failed & 10 \\
\hline \multicolumn{2}{|l|}{ Disease status at transplant } \\
\hline$P R$ & 10 \\
\hline Complete remission & 14 \\
\hline Refractory/progression & 3 \\
\hline PR prior second transplant & $1^{1}$ \\
\hline \multicolumn{2}{|l|}{ Conditioning intensity } \\
\hline Myeloablative & 11 \\
\hline Reduced intensity & $17^{1}$ \\
\hline \multicolumn{2}{|l|}{ Conditioning regimen ${ }^{2}$} \\
\hline $\begin{array}{l}\text { Total body irradiation containing } \\
\text { regimen }\end{array}$ & 15 \\
\hline$\geq 8$ grays & 8 \\
\hline 2 grays & 7 \\
\hline Chemotherapy alone & $13^{1}$ \\
\hline \multicolumn{2}{|l|}{ Type of donor } \\
\hline HLA-identical sibling & 10 \\
\hline Unrelated donor & $18^{1}$ \\
\hline \multicolumn{2}{|l|}{ Source of stem cells } \\
\hline Bone marrow & 4 \\
\hline Peripheral blood stem cells & $20^{1}$ \\
\hline Cord blood & 4 \\
\hline $\begin{array}{l}\text { Median calendar year of } \\
\text { transplant (range) }\end{array}$ & $2009(2000-2013)$ \\
\hline
\end{tabular}

PR, partial response.

${ }^{1}$ One pt received a second RIC allotransplant.

${ }^{2}$ Defined according to Bacigalupo et al. (17).

Table 2 Pretransplant status response and the best response posttransplant

\begin{tabular}{lr}
\hline $\mathrm{PR} \rightarrow \mathrm{CR}$ & 7 \\
Progression or refractory $\rightarrow \mathrm{CR}$ & 2 \\
$\mathrm{CR} \rightarrow \mathrm{CR}$ & 12 \\
$\mathrm{PR} \rightarrow \mathrm{PR}$ & 4 \\
$\mathrm{CR} \rightarrow$ not evaluable & 1 \\
Progression $\rightarrow$ not evaluable & 1 \\
$\mathrm{CR} \rightarrow$ early relapse & 1
\end{tabular}

$\mathrm{PR}$, partial response; $\mathrm{CR}$, complete remission.

${ }^{1}$ One pt received a second RIC allotransplant. 
Figure 1 (A) OS and PFS, (B) cumulative incidence (Cl) of relapse, (C) $\mathrm{Cl}$ of non relapse mortality, and (D) $\mathrm{Cl}$ of acute GHVD II-IV following allogeneic HSCT. HSCT, hematopoietic stem cell transplantation; OS, overall survival; PFS, progression-free survival.
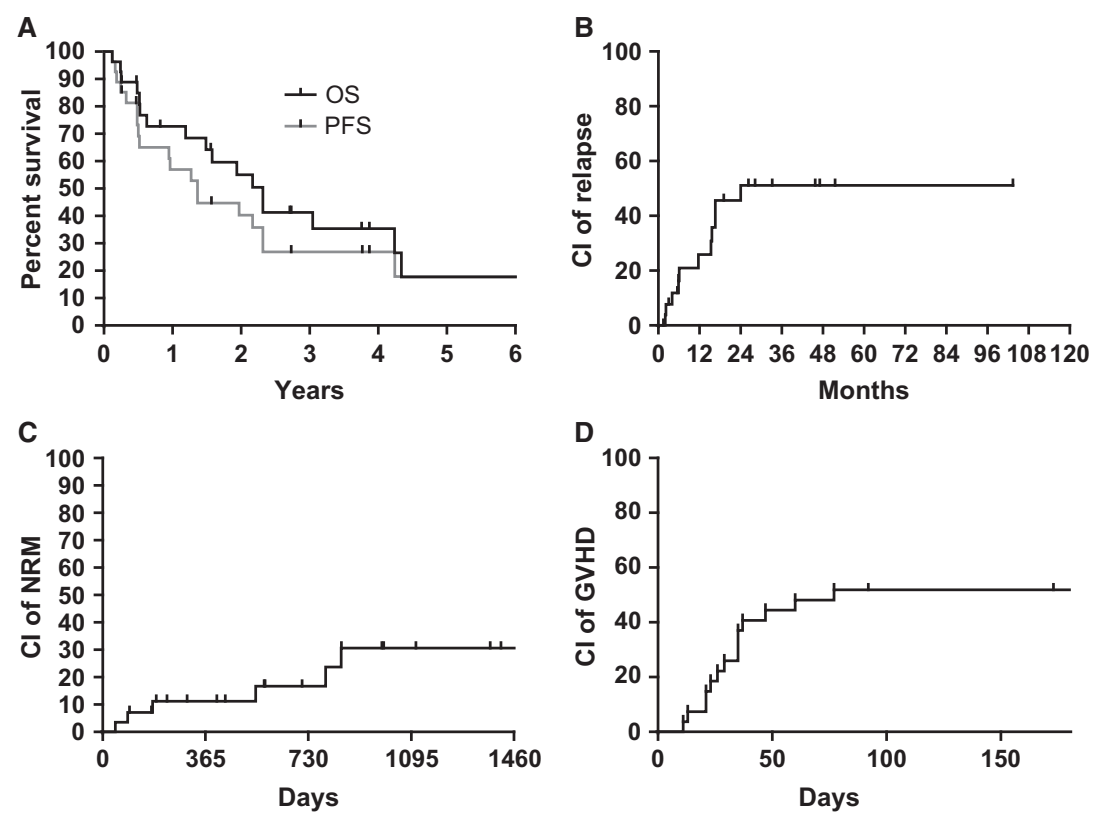

Following transplantation, as best response, 21 patients were in CR1. For two patients, response could not be determined due to early TRM. The pretransplant status and posttransplant best response are summarized on Table 2. After HSCT, engraftment was achieved in $96 \%$ of the patients. With a median follow-up for surviving patients of 33 (range, 6-103) months, 10 patients are still alive all in CR. OS and PFS estimates at $3 \mathrm{yr}$ were $36 \%$ (95\% CI: 17-54\%) and 26\% (95\% CI: 14-45\%), respectively (Fig. 1A). Seventeen patients died, six from transplant-related causes (namely four GVHD, one infection, one cardiac toxicity) eight due to disease progression, one from neoplastic pericarditis, and two from unknown reason.

At last follow-up, the relapse incidence was $47 \%$ (Fig. 1B). Relapse was observed at a median of 11.7 (range, 2-24) months after HSCT. Among the 11 patients who relapsed, the relapse was observed beyond $1 \mathrm{yr}$ for five of them. Ten patients were retreated for post-transplantation relapse. Details of salvage therapy are given on Table 3. Overall cumulative incidence of TRM was $31 \%$ at $3 \mathrm{yr}$ (Fig. 1C). Donor lymphocyte infusions (DLI) were performed in six patients (five for disease relapse and one for mixed donor chimerism). It was efficient in only two cases: a CR was obtained for one patient who unfortunately developed a grade 4 acute digestive GVHD and died from pulmonary carcinoma; mixed chimerism was corrected for the other patient.

Six patients $(22 \%)$ had a prolonged survival beyond 36 months. One died from unknown reason at 51 months from transplantation but not due to relapse, and another one died at 53 months because of relapse (the relapse was treated with alemtuzumab and DLI).

Fifteen patients developed acute GVHD (grade I in three patients, grade II in eight patients, grade III in three patients, and grade IV in one patient). The cumulative incidence of grade II-IV GVHD was $51 \%$ (95\% CI: $32-$ $68 \%$, Fig. 1D). Chronic GVHD was observed in $11(40 \%)$ of the patients, with six having a limited form and five an extensive form.

In the univariate analysis, we could not find any factor associated with improved OS. Regarding the conditioning regimen, we found a trend for better OS and PFS for those who received TBI at 8 or 10 or 12 Gy in the conditioning regimen $(P=0.088 ; P=0.070)$.

Table 3 Treatment of relapse, 1 was not retreated because of infection

\begin{tabular}{ll}
\hline $1 \mathrm{pt}$ & $\begin{array}{c}\text { Alemtuzumab } \rightarrow \text { failure, lenalidomide } \rightarrow \text { failure, } \\
\text { bendamustine } \rightarrow \text { failure }\end{array}$ \\
$1 \mathrm{pt}$ & Lenalidomide and radiotherapy $\rightarrow$ PR \\
$1 \mathrm{pt}$ & Alemtuzumab and $3 \mathrm{DLI} \rightarrow \mathrm{PR}$ and progressed \\
$1 \mathrm{pt}$ & Alemtuzumab and $2 \mathrm{DLI} \rightarrow$ CR complicated by \\
& an acute grade IV GVHD (died \\
& from pulmonary carcinoma) \\
$1 \mathrm{pt}$ & Pentostatin and DLI $\rightarrow$ CR of short duration, \\
& retreated with bendamustine and \\
& lenalidomide $\rightarrow$ failure \\
$1 \mathrm{pt}$ & A short course of alemtuzumab $\rightarrow$ failure, 2 cycles \\
& of pentostatin $\rightarrow$ failure \\
$1 \mathrm{pt}$ & Alemtuzumab and 1 DLI $\rightarrow$ failure \\
$1 \mathrm{pt}$ & DLI $\rightarrow$ failure \\
$1 \mathrm{pt}$ & Pentostatin $\rightarrow$ failure \\
$1 \mathrm{pt}$ & Received a second allogeneic transplantation, \\
& progressed, received \\
& pentostatin $\rightarrow$ failure
\end{tabular}

CR, complete remission; DLI, donor lymphocyte infusions; GVHD, graft-versus-host disease; PR, partial response. 
Table 4 Registry reports on allogeneic transplantation for T-PLL

\begin{tabular}{llllll}
\hline Database & Patients $(n)$ & RIC $(\%)$ & \% CR at transplant & Year of transplant & Outcome (median) \\
\hline CIBMTR (13) & 21 & Not available & Not available & $1995-2006$ & PFS $=5.1$ months \\
EBMT (14) & 41 & 32 & 26 & $2004(1995-2006)$ & OS $=12$ months \\
& 27 & 60 & 52 & $2009(2000-2013)$ & PFS $=10$ months \\
SFGM-TC & & & & OS $=26$ months \\
& & & & PFS $=16.5$ months
\end{tabular}

CR, complete remission; OS, overall survival; PFS, progression-free survival; T-PLL, T-prolymphocytic leukemia.

\section{Discussion}

T-PLL is a rare disease for which treatment remains very disappointing and outcome very dismal. HSCT has been proposed to improve leukemic control and OS, and several case reports suggested a possible benefit from an immune graft-versus-leukemia (GVL) effect (8-14).

Two recent registry analyses of patients treated with allogeneic HSCT have been reported $(15,16)$. The CIBMTR registry included both $\mathrm{B}$ - and T-cell PLL with limited information on the group of 21 patients with T-PLL. The median PFS for these patients was 5.1 months with a relatively short median follow-up. The EBMT and the Royal Marsden Consortium database reported at $3 \mathrm{yr}$ an OS and relapse-free survival of $21 \%$ and $19 \%$, respectively (14). This retrospective analysis included 41 patients transplanted at a median year of 2004 , with more than $50 \%$ receiving a standard myeloablative conditioning regimen.

In the current series, OS and PFS were, respectively, 36\% and $26 \%$ at $3 \mathrm{yr}$. A number of reasons may account for the difference between these results. The current study was performed more recently (median year of transplant: 2009), with more than half of the patients receiving a reducedintensity conditioning regimen (Table 4). We could not find a prognostic factor associated with a better outcome, and this might be due to the small number of heterogeneous patients analyzed. A large proportion of patients had received alemtuzumab subcutaneously either prior transplantation or for post-transplantation relapse. Dearden et al. (6) reported a better efficacy of alemtuzumab delivered intravenously. All patients treated in this series received alemtuzumab subcutaneously mainly for practical reason and better tolerance.

Nevertheless, despite an encouraging response rate, the current analysis confirms that relapse after HSCT remains a matter of concern, even late after HSCT. Hopfinger et al. (7) reported seven long-term survivors (median OS 49.6 months) among the 25 patients treated with the FMC regimen followed by alemtuzumab, five of them were retreated for late relapse. Dearden et al. (6) reported an OS at 48 months at $37 \%$ for those who received alemtuzumab iv as first-line treatment, some of them having been transplanted. In our series, $22 \%$ had a prolonged survival beyond 36 months.
Therefore, appropriate and close follow-up must be maintained to detect such relapse. Additionally, preemptive therapy (early DLI) should be considered to enhance the GVL effect.

We conclude that HSCT may lead to long-term survival in patients with T-PLL following induction treatment. Given the rarity of this disease, an international collaborative effort is needed to further clarify the role of allogeneic transplantation in this devastating disease.

\section{Acknowledgements}

The authors would like to thank members of the Société Française de Greffe de Moelle et de Thérapie Cellulaire (SFGM-TC), the SFGM-TC data manager Nicole Raus, and all collaborators who contributed to patient recruitment, data registration, and quality control.

\section{Conflict of interest}

The authors have no conflict of interest.

\section{References}

1. Matutes E, Brito-Babapulle V, Swansbury J, Ellis J, Morilla R, Dearden C, Sempere A, Catovsky D. Clinical and laboratory features of 78 cases of T-prolymphocytic leukemia. Blood 1991;78:3269-74.

2. Dearden CE. How I treat prolymphocytic leukemia. Blood 2012;120:538-51.

3. Garand R, Goasguen J, Brizard A, et al. Indolent course as a relatively frequent presentation in T-prolymphocytic leukaemia. $\mathrm{Br} \mathrm{J}$ Haematol 1998;103:488-94.

4. Ginaldi L, De Martinis M, Matutes E, Farahat N, Morilla R, Dyer MJ, Catovsky D. Levels of expression of CD52 in normal and leukemic B and T cells: correlation with in vivo therapeutic responses to Campath-1H. Leuk Res 1998;22: 185-91.

5. Dearden CE, Matutes E, Cazin B, et al. High remission rate in T-cell prolymphocytic leukemia with CAMPATH-1H. Blood 2001;98:1721-6.

6. Dearden CE, Khot A, Else M, Hamblin M, Grand E, Roy A, Hewamana S, Matutes E, Catovsky D. Alemtuzumab therapy in T-cell prolymphocytic leukemia: comparing efficacy in a 
series treated intravenously and a study piloting the subcutaneous route. Blood 2011;118:5799-802.

7. Hopfinger G, Busch R, Pflug N, et al. Sequential chemoimmunotherapy of fludarabine, mitoxantrone, and cyclophosphamide induction followed by alemtuzumab consolidation is effective in T-cell prolymphocytic leukemia. Cancer 2013;119:2258-67.

8. Collins RH, Piñeiro LA, Agura ED, Fay JW. Treatment of T prolymphocytic leukemia with allogeneic bone marrow transplantation. Bone Marrow Transplant 1998;21:627-8.

9. Krishnan B, Else M, Tjonnfjord GE, et al. Stem cell transplantation after alemtuzumab in T-cell prolymphocytic leukaemia results in longer survival than after alemtuzumab alone: a multicentre retrospective study. Br J Haematol 2010;149:90710.

10. Garderet L, Bittencourt H, Kaliski A, Daniel M, Ribaud P, Socié G, Gluckman E. Treatment of T-prolymphocytic leukemia with nonmyeloablative allogeneic stem cell transplantation. Eur J Haematol 2001;66:137-9.

11. de Lavallade H, Faucher C, Fürst S, et al. Allogeneic stem cell transplantation after reduced-intensity conditioning in a patient with T-cell prolymphocytic leukemia: graft-versustumor effect and long-term remission. Bone Marrow Transplant 2006;37:709-10.
12. Tanimoto TE, Hirano A, Nagafuji K, et al. Mismatched unrelated cord blood transplantation in a patient with T-cell prolymphocytic leukemia. Leukemia 2005;19:679-81.

13. Murase K, Matsunaga T, Sato T, et al. Allogeneic bone marrow transplantation in a patient with T-prolymphocytic leukemia with small-intestinal involvement. Int J Clin Oncol 2003;8:391-4.

14. Kruspe RC, Ashraf KK, Foran JM, Salzman DE, Reddy VV, Vaughan WP. Successful treatment of T-cell prolymphocytic leukemia with full-intensity conditioning followed by matched unrelated donor allogeneic stem cell transplantation. Clin Adv Hematol Oncol 2007;5:882-4.

15. Kalaycio ME, Kukreja M, Woolfrey AE, et al. Allogeneic hematopoietic cell transplant for prolymphocytic leukemia. Biol Blood Marrow Transplant 2010;16:543-7.

16. Wiktor-Jedrzejczak W, Dearden C, de Wreede 1, et al. Hematopoietic stem cell transplantation in T-prolymphocytic leukemia: a retrospective study from the European Group for Blood and Marrow Transplantation and the Royal Marsden Consortium. Leukemia 2011;26:972-6.

17. Bacigalupo A, Ballen K, Rizzo D, et al. Defining the intensity of conditioning regimens: working definitions. Biol Blood Marrow Transplant 2009;15:1628-33. 